\title{
REFLECTIVE SUBCATEGORIES
}

\author{
JUAN RADA \\ Departamento de Matemáticas, Universidad de los Andes, 5101 Mérida, Venezuela \\ e-mail: juanrada@ciens.ula.ve \\ MANUEL SAORÍN and ALBERTO DEL VALLE \\ Departamento de Matemáticas, Universidad de Murcia, Aptdo. 4021, Espinardo 30.100, Murcia, Spain \\ e-mail:msaorinc@fcu.um.es, alberto@fcu.um.es
}

(Received 15 June, 1998)

Dedicated to Kent Fuller on his 60th Anniversary

\begin{abstract}
Given a full subcategory $\mathcal{F}$ of a category $\mathcal{A}$, the existence of left $\mathcal{F}$ approximations (or $\mathcal{F}$-preenvelopes) completing diagrams in a unique way is equivalent to the fact that $\mathcal{F}$ is reflective in $\mathcal{A}$, in the classical terminology of category theory.

In the first part of the paper we establish, for a rather general $\mathcal{A}$, the relationship between reflectivity and covariant finiteness of $\mathcal{F}$ in $\mathcal{A}$, and generalize Freyd's adjoint functor theorem (for inclusion functors) to not necessarily complete categories. Also, we study the good behaviour of reflections with respect to direct limits. Most results in this part are dualizable, thus providing corresponding versions for coreflective subcategories.

In the second half of the paper we give several examples of reflective subcategories of abelian and module categories, mainly of subcategories of the form Copres $(M)$ and Add $(M)$. The second case covers the study of all covariantly finite, generalized Krull-Schmidt subcategories of $\operatorname{Mod}_{R}$, and has some connections with the "puresemisimple conjecture".
\end{abstract}

1991 Mathematics Subject Classification. 18A40, 16D90, 16E70

1. Introduction. In recent times, there has been strong interest in the study of (pre)envelopes and (pre)covers by an arbitrary class $\mathcal{F}$ of modules (cf. [11], [25], [2], [26]). One of the open problems there is to find when every module has an $\mathcal{F}$-envelope completing diagrams in a unique way. Results for the case when $\mathcal{F}$ is the class of projective or flat modules can be found in [2], [10], [12]. For an arbitrary class $\mathcal{F}$ of objects of a category $\mathcal{A}$, the existence of an $\mathcal{F}$-envelope completing diagrams uniquely for every object of $\mathcal{A}$ is equivalent to the fact that $\mathcal{F}$ is a reflective subcategory of $\mathcal{A}$ in the classical terminology of category theory (see, e.g., [31]). The goal of this work is twofold. On one side, we shall show that Freyd's adjoint functor theorem, in the case of an inclusion functor, is closely related to the notion of a covariantly finite subcategory introduced by Auslander's school, which also allows us in this case to extend Freyd's theorem to noncomplete categories (see Theorem 3.1 and its corollaries). By using that, as a second objective, we shall give a good supply of examples of reflective and coreflective subcategories, mainly in module and abelian categories.

After introducing our notation and terminology, Section 3 contains the already mentioned generalizations of Freyd's theorem which allow applications to noncomplete categories; also, given a locally finitely presented abelian category, we give criteria for a subcategory consisting of direct limits of its finitely presented objects to 
be reflective. In Section 4 we give applications of the previous results, with special attention to subcategories of an abelian category $\mathcal{A}$ of the form Copres $(M)$ for an object $M$ of $\mathcal{A}$. Finally, Section 5 studies the reflective subcategories of $\operatorname{Mod}_{R}$ which are generalized Krull-Schmidt (see definition in Section 5).

2. Notation and terminology. Let $\mathcal{A}$ be any category and $\mathcal{F}$ any class of objects of $\mathcal{A}$ (all classes of objects in a category are considered to be closed under isomorphisms, and we shall usually identify $\mathcal{F}$ with the full subcategory of $\mathcal{A}$ whose objects are those of $\mathcal{F}$ ). An object of $\mathcal{F}$ will be called an $\mathcal{F}$-object, and an $\mathcal{F}$ morphism will be a morphism between $\mathcal{F}$-objects.

An $\mathcal{F}$-preenvelope of the $\mathcal{A}$-object $A$ is a morphism $\lambda: A \rightarrow F$ with $F \in \mathcal{F}$ such that, for any other morphism $\lambda^{\prime}: A \rightarrow F^{\prime}$ with $F^{\prime} \in \mathcal{F}$, there exists a morphism $\mu: F \rightarrow F^{\prime}$ such that $\mu \circ \lambda=\lambda^{\prime}$. This means that the map $\mathcal{A}\left(F, F^{\prime}\right) \rightarrow \mathcal{A}\left(A, F^{\prime}\right)$ induced by $\lambda$ is surjective for any $\mathcal{F}$-object $F^{\prime}$. An $\mathcal{F}$-preenvelope of $A$ is also called a left $\mathcal{F}$-approximation of $A$, and the class $\mathcal{F}$ is said to be covariantly finite when every $\mathcal{A}$-object has an $\mathcal{F}$-preenvelope. The $\mathcal{F}$-preenvelope $\lambda: A \rightarrow F$ is said to be an $\mathcal{F}$-envelope (or a minimal left $\mathcal{F}$-approximation) when it is left minimal i.e. when the preimages of $\lambda$ via $\mathcal{A}(F, F) \rightarrow \mathcal{A}(A, F)$ are all automorphisms of $F$. The dual concepts are $\mathcal{F}$-precover (or right $\mathcal{F}$-approximation), contravariantly finite class and $\mathcal{F}$ cover (or minimal right $\mathcal{F}$-approximation).

An $\mathcal{F}$-(pre)envelope $A \rightarrow F$ for which the induced maps $\mathcal{A}\left(F, F^{\prime}\right) \rightarrow \mathcal{A}\left(A, F^{\prime}\right)$ (with $F^{\prime} \in \mathcal{F}$ ) are all bijective is called an $\mathcal{F}$-reflection of $A$ ([18], [24]; it is called an $\mathcal{F}$-envelope which complete diagrams in a unique way in [2]). A full subcategory $\mathcal{F}$ of $\mathcal{A}$ is said to be reflective in $\mathcal{A}([\mathbf{1 8}]$, [24]) when the inclusion functor $U: \mathcal{F} \hookrightarrow \mathcal{A}$ has a left adjoint $F: \mathcal{A} \rightarrow \mathcal{F}$ (called a reflector for $\mathcal{F}$ ). In this case the unit of the adjunction $\eta: 1_{\mathcal{A}} \rightarrow U \circ F$ gives an $\mathcal{F}$-reflection $\eta_{A}$ for each $\mathcal{A}$-object $A$ and, conversely, when each $\mathcal{A}$-object has an $\mathcal{F}$-reflection, it is clear how to define a reflector for $\mathcal{F}$. The dual concepts are $\mathcal{F}$-coreflection and coreflective subcategory.

The class $\mathcal{F}$ is said to be locally initially small in $\mathcal{A}$ [26] if, for every $\mathcal{A}$-object $A$, there exists a set $\mathcal{F}_{A} \subseteq \mathcal{F}$ such that every morphism $A \rightarrow F$ with $F \in \mathcal{F}$ factors through a direct product of modules in $\mathcal{F}_{A}$. The dual concept is that of a locally finally small class. The class $\mathcal{F}$ is said to be closed for retracts (in $\mathcal{A}$ ) if, whenever $F \in \mathcal{F}$ and $F \rightarrow F^{\prime}$ is a retraction in $\mathcal{A}$, we have $F^{\prime} \in \mathcal{F}$. It is easy to see that, if $\mathcal{F}$ is reflective in $\mathcal{A}$ (or, more generally, if every object of $\mathcal{A}$ has an $\mathcal{F}$-envelope), then $\mathcal{F}$ is closed for retracts.

Most of our results are given for well-powered categories, i.e., categories in which the subobjects of every given object form a set. Following [9], an object $X$ of a preadditive category $\mathcal{A}$ with direct limits will be called finitely presented when the functor $\operatorname{Hom}_{\mathcal{A}}(X,-)$ preserves direct limits. When the finitely presented objects of $\mathcal{A}$ form a skeletally small class $\mathrm{fp}(\mathcal{A})$ of generators, we shall say that $\mathcal{A}$ is locally finitely presented. In that case, the finitely generated objects of $\mathcal{A}$ are just the homomorphic images of morphisms with finitely presented domain. When, moreover, every finitely generated subobject of a finitely presented one is again finitely presented, we shall say that $\mathcal{A}$ is locally coherent. For a class $\mathcal{F}$ of objects of $\operatorname{fp}(\mathcal{A})$ we shall denote by $\overrightarrow{\mathcal{F}}$ the class of all objects of $\mathcal{A}$ that are (isomorphic to) direct limits of objects in $\mathcal{F}$.

Following [30], a coperfect object in a locally finitely presented category is one that satisfies DCC on finitely generated subobjects. When every object of $\mathcal{A}$ is coperfect, we say that $\mathcal{A}$ is a coperfect category. 
All our rings are associative with identity, and all our modules are unital. We denote by $\operatorname{Mod}_{R}\left(\right.$ resp. $\left.\bmod _{R}\right)$ the category of all (resp. all finitely presented) right $R$-modules. We write pd $\left(M_{R}\right)$ for the projective dimension of the right $R$-module $M_{R}$, and $\operatorname{rg} D(R)$ (resp. $\operatorname{lgD}(R), \mathrm{wD}(R)$ ) for the right global (resp. left global, weak global) dimension of the ring $R$.

\section{Characterizations of reflective subcategories.}

Definition 3.1. Let $\mathcal{A}$ be any category and let $\mathcal{F}$ be a full subcategory of $\mathcal{A}$.

We say that (finite) products of $\mathcal{F}$-objects exist in $\mathcal{A}$ when any (finite) family of $\mathcal{F}$-objects has a product in $\mathcal{A}$.

We say that equalizers of $\mathcal{F}$-morphisms exist in $\mathcal{A}$ when any pair of $\mathcal{F}$-morphisms with the same domain and codomain has an equalizer in $\mathcal{A}$. If $\mathcal{A}$ has zero morphisms we get, as a particular case, the definition that kernels of $\mathcal{F}$-morphisms exist in $\mathcal{A}$.

When equalizers of $\mathcal{F}$-morphisms exist in $\mathcal{A}$ and, for any $\mathcal{F}$-object $F$, every downward directed family $\left\{\operatorname{Eq}\left(f_{i}, g_{i}\right)\right\}_{i \in I}$ (indexed by a set) of equalizers of pairs of $\mathcal{F}$-morphisms with domain $F$ and common codomain has the property that $\cap_{i \in I} \operatorname{Eq}\left(f_{i}, g_{i}\right)$ exists in $\mathcal{A}$ and, moreover, $\cap_{i \in I} \operatorname{Eq}\left(f_{i}, g_{i}\right)=\operatorname{Eq}(f, g)$ for some pair of $\mathcal{F}$-morphism $f, g$ with domain $F$ and common codomain, then we shall say that strong equalizers of $\mathcal{F}$-morphisms exist in $\mathcal{A}$. Similarly, one gets the definition that strong kernels of $\mathcal{F}$-morphisms exist in $\mathcal{A}$.

Before giving our results, we present several examples of classes with the properties defined above.

EXAmple 3.1. (1) Let $\mathcal{A}$ be a complete category and $\mathcal{F}$ be any class of objects in $\mathcal{A}$. Then products of $\mathcal{F}$-objects and equalizers of $\mathcal{F}$-morphisms always exist in $\mathcal{A}$.

(2) Let $\mathcal{A}$ be a locally coherent abelian category; then, for every class $\mathcal{F}$ of objects in $\mathrm{fp}(\mathcal{A})$, kernels of $\mathcal{F}$-morphisms exist in $\mathrm{fp}(\mathcal{A})$. Moreover, if $\mathcal{F}$ has products in $\mathcal{A}$ and is closed for retracts and finite products, then strong kernels of $\mathcal{F}$ morphisms exist in $\mathrm{fp}(\mathcal{A})$ provided that the following condition is satisfied: for every morphism $f: F \rightarrow \prod_{i \in I} F_{i}$ with $F$ and each $F_{i}$ in $\mathcal{F}$, there exists a finite subset $J$ of $I$ such that $\operatorname{Ker} f=\operatorname{Ker}\left(\pi_{J} \circ f\right)$, where $\pi_{J}: \prod_{i \in I} F_{i} \rightarrow \prod_{i \in J} F_{i}$ is the canonical projection. In particular, this happens when $\mathcal{F}$ consists of coperfect objects.

(3) A particular case of (2) is the following. If $R$ is a right coherent and left perfect ring, then for every class $\mathcal{F} \subseteq \bmod _{R}$ closed for direct summands and finite direct sums, strong kernels of $\mathcal{F}$-morphisms exist in $\bmod _{R}$.

(4) If $\mathcal{A}$ is a locally finitely presented abelian category and $\mathcal{F} \subseteq \mathrm{fp}(\mathcal{A})$ is a class of objects of finite length all whose composition factors are finitely presented, then strong kernels of $\mathcal{F}$-morphisms exist in $\operatorname{fp}(\mathcal{A})$.

TheOREM 3.1. Let $\mathcal{A}$ be a well-powered category and let $\mathcal{F}$ be a full subcategory of $\mathcal{A}$ such that equalizers of $\mathcal{F}$-morphisms and intersections of them exist in $\mathcal{A}$. The following assertions are equivalent:

(a) $\mathcal{F}$ is reflective in $\mathcal{A}$;

(b) $\mathcal{F}$ is covariantly finite in $\mathcal{A}$ and closed for intersections of equalizers of $\mathcal{F}$ morphisms. 
Proof. (a) $\Rightarrow$ (b). This follows by [18, (36.13)].

(b) $\Rightarrow$ (a). Let $\lambda: A \rightarrow F$ be an $\mathcal{F}$-preenvelope of an arbitrary $\mathcal{A}$-object $A$ and consider the class $\Omega$ of all pairs of morphisms $f, g: F \rightarrow F^{\prime}$ with $F^{\prime} \in \mathcal{F}$ and $f \circ \lambda=g \circ \lambda$. Since $\mathcal{A}$ is well-powered, we can fix a family $\left\{f_{i, g_{i}}: F \rightarrow F_{i} \mid i \in I\right\}$ (indexed by a set $I$ ) of elements of $\Omega$ such that the equalizer of every pair of morphisms in $\Omega$ equals $\operatorname{Eq}\left(f_{i}, g_{i}\right)$ for some $i \in I$. Let now $e: E \rightarrow F$ be the intersection of the family $\left\{\operatorname{Eq}\left(f_{i}, g_{i}\right) \mid i \in I\right\}$; by hypothesis we have $E \in \mathcal{F}$, and by construction there exists a unique morphism $\mu: A \rightarrow E$ such that $e \circ \mu=\lambda$. Then it is clear that $\mu$ is an $\mathcal{F}$-preenvelope of $A$, and we shall prove that it is indeed an $\mathcal{F}$ reflection, which will finish the proof of the theorem. For this, we start by noting that, whenever $f, g: F \rightarrow F^{\prime}$ is a pair of $\mathcal{F}$-morphisms with $f \circ \lambda=g \circ \lambda$ and $e^{\prime}: E^{\prime} \rightarrow F$ is its equalizer, there is a unique monomorphism $u: E \rightarrow E^{\prime}$ such that $e=e^{\prime} \circ u$. Now we check that $e$ is a section: the preenveloping condition of $\lambda$ gives a morphism $p: F \rightarrow E$ with $\mu=p \circ \lambda$, and hence

$$
e \circ p \circ \lambda=e \circ \mu=\lambda=1_{F} \circ \lambda \text {. }
$$

If $e^{\prime}: E^{\prime} \rightarrow F$ is the equalizer of $1_{F}$ and $e \circ p$ then there is a monomorphism $u: E \rightarrow E^{\prime}$ with $e=e^{\prime} \circ u$, and therefore

$$
e \circ 1_{E}=e=1_{F} \circ e=1_{F} \circ e^{\prime} \circ u=e \circ p \circ e^{\prime} \circ u=e \circ p \circ e ;
$$

as $e$ is monic this implies that $p \circ e=1_{E}$, as claimed. Now suppose that $h, h^{\prime}: E \rightarrow G$ are $\mathcal{F}$-morphisms with $h \circ \mu=h^{\prime} \circ \mu$. Then $h \circ p \circ \lambda=h^{\prime} \circ p \circ \lambda$, and if $e^{\prime \prime}: E^{\prime \prime} \rightarrow F$ is the equalizer of $h \circ p$ and $h^{\prime} \circ p$ then there is a monomorphism $v: E \rightarrow E^{\prime \prime}$ with $e=e^{\prime \prime} \circ v$, and therefore

$$
h=h \circ p \circ e=h \circ p \circ e^{\prime \prime} \circ v=h^{\prime} \circ p \circ e^{\prime \prime} \circ v=h^{\prime} \circ p \circ e=h^{\prime} .
$$

The following result generalizes Freyd's adjoint functor theorem for inclusion functors (see [24, p. 117, Theorem 2]). Note that the condition " $\mathcal{F}$ is locally initially small" is more general than the "solution set condition" of the above cited result; indeed, if $\mathcal{R}$ is any ring and $\mathcal{F}$ is the class of all flat right $R$-modules, then $\mathcal{F}$ is locally initially small in $\operatorname{Mod}_{R}$ [26, Proposition 2.8], but it has the solution set condition if and only if $R$ is left coherent [11, Proposition 5.1].

COROLlaRY 3.2. Let $\mathcal{A}$ be a well-powered category and let $\mathcal{F}$ be a full subcategory of $\mathcal{A}$ such that products of $\mathcal{F}$-objects and equalizers of $\mathcal{F}$-morphisms exist in $\mathcal{A}$. The following assertions are equivalent:

(a) $\mathcal{F}$ is reflective in $\mathcal{A}$;

(b) $\mathcal{F}$ is covariantly finite in $\mathcal{A}$ and closed for equalizers;

(c) $\mathcal{F}$ is locally initially small in $\mathcal{A}$ and closed for equalizers and products.

Proof. (a) $\Rightarrow$ (b) follows as before, (b) $\Longleftrightarrow$ (c) is proven as in [26, Theorem 3.3] (note that $\mathcal{F}$ is closed for retracts since it is closed for equalizers), and (b,c) $\Rightarrow$ (a) follows from [18, (18.23)] and Theorem 3.1.

Next we give a third partial version of Theorem 3.1 that is useful to deal with noncomplete categories. 
COROLlary 3.3. Let $\mathcal{A}$ be a well-powered category and let $\mathcal{F}$ be a full subcategory of $\mathcal{A}$ such that finite products of $\mathcal{F}$-objects and strong equalizers of $\mathcal{F}$-morphisms exist in $\mathcal{A}$. Then the following assertions are equivalent:

(a) $\mathcal{F}$ is reflective in $\mathcal{A}$;

(b) $\mathcal{F}$ is covariantly finite in $\mathcal{A}$ and closed for equalizers.

Proof. We only need to prove $(b) \Rightarrow(a)$, and for this one can adapt the proof of Theorem 3.1 after showing that, given $\left\{f_{i}, g_{i}: F \rightarrow F_{i} \mid i \in I\right\}$ as there, $\cap_{i \in I} \operatorname{Eq}\left(f_{i}, g_{i}\right)$ exists and belongs to $\mathcal{F}$. Now, by the hypotheses, it is enough to show that $\left\{\mathrm{Eq}\left(f_{i}, g_{i}\right) \mid i \in I\right\}$ is a downward directed family. For, given $i, j \in I$, let $F^{\prime}$ be the product of $F_{i}$ and $F_{j}$ and let $f$ (resp.g) be the morphism $F \rightarrow F^{\prime}$ induced by $f_{i}, f_{j}\left(\right.$ resp. $\left.g_{i}, g_{j}\right)$. By hypothesis, there exists $k \in I$ such that $\operatorname{Eq}(f, g)=\operatorname{Eq}\left(f_{k}, g_{k}\right)$, and this is a subobject of both $\operatorname{Eq}\left(f_{i}, g_{i}\right)$ and $\operatorname{Eq}\left(f_{j}, g_{j}\right)$.

Remark 3.1. (1) Corollary 3.2 applies to Example 3.1.(1), showing that a full subcategory of a well-powered complete category $\mathcal{A}$ is reflective in $\mathcal{A}$ if and only if it is locally initially small and closed for products and equalizers.

(2) Corollary 3.3 applies to Example 3.1(2-4), showing for instance that if $\mathcal{A}$ is a locally coherent abelian category and $\mathcal{F} \subseteq \mathrm{fp}(\mathcal{A})$ is a subcategory consisting of coperfect objects, then $\mathcal{F}$ is reflective in $\mathrm{fp}(\mathcal{A})$ if and only if $\mathcal{F}$ is covariantly finite in $\mathrm{fp}(\mathcal{A})$ and closed for kernels and finite direct sums.

REMARK 3.2. The above results admit straightforward dualizations which are left to the reader.

For suitable classes $\mathcal{F}$, the uniqueness in the completion of diagrams allows us to construct, from an $\mathcal{F}$-reflection for each member of a direct system, an $\mathcal{F}$-reflection for the direct limit of the system. This fact is exploited in the following result.

Proposition 3.4. Let $\mathcal{A}$ be a locally finitely presented category, and let $\mathcal{F} \subseteq \operatorname{fp}(\mathcal{A})$ be a class of objects closed for retracts. Then $\mathcal{F}$ is reflective in $\mathrm{fp}(\mathcal{A})$ if and only if $\overrightarrow{\mathcal{F}}$ is reflective in $\mathcal{A}$.

Proof. (a) $\Rightarrow(\mathrm{b})$. First note that, if $X \in \mathrm{fp}(\mathcal{A})$ and $\lambda: X \rightarrow F$ is an $\mathcal{F}$-(pre)envelope, then it is an $\overrightarrow{\mathcal{F}}$-(pre)envelope. Moreover, if it is an $\mathcal{F}$-reflection then it is also an $\overrightarrow{\mathcal{F}}$-reflection. To see this it is enough to show that, if $\bar{F} \in \overrightarrow{\mathcal{F}}$ (say $\bar{F}=\lim _{\rightarrow} F_{i}$ with each $F_{i} \in \mathcal{F}$ and canonical maps $\phi_{i}: F_{i} \rightarrow \bar{F}$ ), then any morphism $\alpha: F \rightarrow \bar{F}$ with $\alpha \circ \lambda=0$ must be zero. By [9, Lemma 1.1.3], there exists an index $i$ and a morphism $\beta: F \rightarrow F_{i}$ with $\beta \circ \lambda=0$ such that $\alpha=\phi_{i} \circ \beta$. Now, the fact that $\lambda$ is an $\mathcal{F}$-reflection gives $\beta=0$ and hence $\alpha=0$. The rest of the proof follows that of [25, Theorem 2.11].

(b) $\Rightarrow$ (a). If $X \in \mathrm{fp}(\mathcal{A})$ and $\lambda: X \rightarrow F$ is an $\overrightarrow{\mathcal{F}}$-reflection, where $F=\lim _{i} F_{i}$ with each $F_{i} \in \mathcal{F}$ and canonical maps $\phi_{i}: F_{i} \rightarrow F$, then $\lambda=\phi_{i} \circ \lambda^{\prime}$ for some $\lambda^{\prime}: X \rightarrow F_{i}$, but in turn this $\lambda^{\prime}$ factors as $\lambda^{\prime}=h \circ \lambda$ and, by the uniqueness in the completion of diagrams, we get $\phi_{i} \circ h=1_{F}$. So, by hypothesis, $F \in \mathcal{F}$ and therefore $\lambda$ is an $\mathcal{F}$ reflection.

REMARK 3.3. (1) We cannot omit the hypothesis that $\mathcal{F}$ is closed for retracts. For example, if $R$ is a von Neumann regular ring and $\mathcal{F}$ is the class of all finitely 
generated free modules, then $\mathcal{F}=\operatorname{Mod}_{R}$ but $\mathcal{F}$ is not reflective in $\bmod _{R}$ unless $R$ is a division ring.

(2) Putting together [9, Theorem 4.2], [22, Theorem 12.3] and results of $[\mathbf{1 5}, \S 7 \&$ ff.], one easily deduces that, in the situation of our Proposition 3.4, if $\mathcal{A}$ is (co)complete and kernels of $\mathcal{F}$-morphisms are finitely presented, then (a-b) hold if and only if $\overrightarrow{\mathcal{F}}$ is closed for products and kernels, if and only if $\overrightarrow{\mathcal{F}}$ is closed for products and $\mathcal{F}$ is closed for kernels.

(3) Proposition 3.4 together with the above remark can be used to characterize coreflectivity of subcategories of $\bmod _{R}$, when $R$ is a ring with self-duality $[1, \S 30]$.

4. Applications. We start by applying the results in Section 3 to some "classical" classes of modules. We write $\operatorname{Inj}_{R}, \operatorname{Proj}_{R}$, Flat $_{R}$, and FPInj ${ }_{R}$ for the classes of all injective, projective, flat and FP-injective right $R$-modules, respectively. Also, $\mathcal{P}^{<\infty}$, $\mathcal{I}^{<\infty}$ and $\mathcal{F}^{<\infty}$ denote the classes of right $R$-modules of finite projective, injective and flat dimension, respectively.

Proposition 4.1. For any ring $R$, the following conditions hold.

(i) $\operatorname{Inj}_{R}$ is reflective in $\operatorname{Mod}_{R}$ iff $\operatorname{Proj}_{R}$ is coreflective in $\operatorname{Mod}_{R}$ iff $R$ is semisimple.

(ii) FPInj $_{R}$ is reflective in $\operatorname{Mod}_{R}$ iff Flat ${ }_{R}$ is coreflective in $\operatorname{Mod}_{R}$ iff $R$ is von Neumann regular.

(iii) $\mathcal{P}^{<\infty}$ is reflective in $\operatorname{Mod}_{R}$ iff $\mathcal{P}^{<\infty}$ is coreflective in $\operatorname{Mod}_{R}$ iff $\mathcal{I}^{<\infty}$ is reflective in $\operatorname{Mod}_{R}$ iff $\mathcal{I}^{<\infty}$ is coreflective in $\operatorname{Mod}_{R}$ iff $\operatorname{rgD}(R)<\infty$

(iv) $\mathcal{F}^{<\infty}$ is reflective in $\operatorname{Mod}_{R}$ iff $\mathcal{F}^{<\infty}$ is coreflective in $\operatorname{Mod}_{R}$ iff $\mathrm{wD}(R)<\infty$.

(v) Flat ${ }_{R}$ is reflective in $\operatorname{Mod}_{R}$ iff $R$ is left coherent and $\mathrm{wD}(R) \leq 2$. [2, Prop. 2.1]

(vi) $\operatorname{Proj}_{R}$ is reflective in $\operatorname{Mod}_{R}$ iff $R$ is right perfect, left coherent, and $\operatorname{rgD}(R) \leq 2$.

(vii) $\operatorname{Inj}_{R}$ is coreflective in $\operatorname{Mod}_{R}$ iff $R$ is right noetherian and $\operatorname{rgD}(R) \leq 2$.

Proof. (i-iv) follow from Corollary 3.2 and its dual, since each of the relevant classes equals $\operatorname{Mod}_{R}$ when it is closed for kernels or cokernels (depending on whether we are considering reflectivity or coreflectivity). By [26, Propositions 2.8 and 2.9], Flat ${ }_{R}$ and $\operatorname{Proj}_{R}$ are always locally initially small classes, so that (v) and (vi) follow again by Corollary 3.2. Finally, if $R$ is right noetherian then $\operatorname{Inj}_{R}$ is a locally finally small class and therefore (vii) follows from the dual of Corollary 3.2.

In the next application we consider a skeletally small preadditive category $\mathcal{C}$, and write $\left(\mathcal{C}^{\mathrm{op}}, \mathrm{Ab}\right)$ for the category of contravariant functors from $\mathcal{C}$ to the category $\mathrm{Ab}$ of abelian groups. A functor $F \in\left(\mathcal{C}^{\mathrm{op}}, \mathrm{Ab}\right)$ is representable if it is naturally isomorphic to $(-, C)=\operatorname{Hom}_{\mathcal{C}}(-, C)$ for some $C \in \mathcal{C}$, and a direct limit of representable functors is said to be flat (see, e.g., [9]).

Proposition 4.2. Let $\mathcal{C}$ be as above, and let $\mathcal{R}($ resp. $\mathcal{F})$ be the class of all representable (resp. flat) functors in $\left(\mathcal{C}^{\mathrm{op}}, \mathrm{Ab}\right)$. Then the following assertions are equivalent:

(a) $\mathcal{C}$ has cokernels;

(b) $\mathcal{R}$ is a reflective subcategory of $\mathrm{fp}\left(\mathcal{C}^{\mathrm{op}}, \mathrm{Ab}\right)$;

(c) $\mathcal{F}$ is a reflective subcategory of $\left(\mathcal{C}^{\mathrm{op}}, \mathrm{Ab}\right)$. 
Proof. Assume that $\mathcal{C}$ has cokernels and let $F \in \mathrm{fp}\left(\mathcal{C}^{\mathrm{op}}, \mathrm{Ab}\right)$; then there exists a morphism $f: C \rightarrow C^{\prime}$ in $\mathcal{C}$ and an exact sequence $(-, C) \stackrel{(-, f)}{\rightarrow}\left(-, C^{\prime}\right) \stackrel{\tau}{\rightarrow} F \rightarrow 0$ in $\left(\mathcal{C}^{\text {op }}, \mathrm{Ab}\right)$. If $g: C^{\prime} \rightarrow C^{\prime \prime}$ is the cokernel of $f$ then $(-, g)$ factors through $\tau$ and, using Yoneda's Lemma, it is easy to see that the morphism $\sigma: F \rightarrow\left(-, C^{\prime \prime}\right)$ such that $(-, g)=\sigma \circ \tau$ is an $\mathcal{R}$-reflection of $F$. This proves (a) $\Rightarrow($ b). The converse is proven along the same lines, and (b) $\Longleftrightarrow$ (c) follows from Proposition 3.4 , since $\mathcal{F}=\overrightarrow{\mathcal{R}}$.

REMARK 4.1. (1) When $\mathcal{C}=\bmod _{R}$ or $\mathcal{C}=\mathrm{FMod}_{R}$ (the class of all finitely generated modules) then $\mathcal{C}$ has cokernels and therefore $\mathcal{F}$ is reflective in $\left(\mathcal{C}^{\mathrm{op}}, \mathrm{Ab}\right)$.

(2) When $\mathcal{C}=\operatorname{proj}_{R}$ (the class of all finitely generated projective modules) then $\left(C^{\text {op }}, \mathrm{Ab}\right)$ and $\operatorname{Mod}_{R}$ are equivalent, and thus in Proposition 4.1.(v) we can add the condition that $\operatorname{proj}_{R}$ has cokernels ([4, Proposition 3]).

(3) Straightforward variations on the above arguments prove that $\mathcal{R}$ is covariantly finite in $\mathrm{fp}\left(\mathcal{C}^{\mathrm{op}}, \mathrm{Ab}\right)$ if and only if $\mathcal{C}$ has pseudo-cokernels (see [9]), and that the subcategory of representable covariant functors is reflective (resp. covariantly finite) in $\mathrm{fp}(\mathcal{C}, \mathrm{Ab})$ if and only if $\mathcal{C}$ has kernels (resp. pseudo-kernels).

In the rest of this section, we let $\mathcal{A}$ be a complete abelian category; for a fixed object $M \in \mathcal{A}$, we say that an object $A \in \mathcal{A}$ is $M$-cogenerated if there exists a monomorphism $A \rightarrow M^{I}$ for some set $I$, and we say that $A$ is $M$-copresented if there exists an exact sequence $0 \rightarrow A \rightarrow M^{I} \rightarrow M^{J}$ for some sets $I, J$. We denote by Cogen $(M)$ and Copres $(M)$ the classes of $M$-cogenerated and $M$-copresented objects in $\mathcal{A}$, respectively, and by $\operatorname{Prod}(M)$ the class of all modules isomorphic to a product of copies of $M$.

Copres $(M)$ can be thought of as the closure of $M$ with respect to products and kernels of morphisms between these products. In view of Corollary 3.2, Copres $(M)$ is always contained in (and should be very close to) the smallest reflective subcategory of $\mathcal{A}$ containing $M$. It seems thus interesting to give necessary and sufficient conditions for Copres $(M)$ to be a reflective subcategory of $\mathcal{A}$. One such condition can be derived from [31, (16.4.7)] (note that Copres $(M)$ is always closed for products), but we give it as a consequence of a more general result which may have independent interest.

Proposition 4.3. Let $\mathcal{A}$ be a complete abelian category, and let $M$ be any object of $\mathcal{A}$. Then Copres $(M)$ is covariantly finite in $\mathcal{A}$. In particular, when $\mathcal{A}$ is wellpowered, Copres $(M)$ is a reflective subcategory of $\mathcal{A}$ if and only if it is closed for kernels.

Proof. We prove that every object $A$ of $\mathcal{A}$ has a Copres $(M)$-preenvelope, and the second part of the statement will then be a consequence of Corollary 3.2.

Let $H=\operatorname{Hom}(A, M)$ and let $\sigma: A \rightarrow M^{H}$ be the morphism induced by the family $\{h\}_{h \in H}$, that is $h=\pi_{h} \circ \sigma$ for every $h \in H$, where $\pi_{h}: M^{H} \rightarrow M$ are the canonical projections. Then it is easy to see that $\sigma$ is a $\operatorname{Prod}(M)$-preenvelope of $A$.

We claim that, if $\alpha: A \rightarrow M^{I}$ is a morphism in $\mathcal{A}$ and $\left(k_{\alpha}, K_{\alpha}\right)$ is the intersection of the kernels of all morphisms $f: M^{I} \rightarrow M$ such that $f \circ \alpha=0$, then $\alpha$ factors through $k_{\alpha}$ and $K_{\alpha} \in$ Copres $(M)$. The first assertion is clear and, if $J=\left\{f: M^{I} \rightarrow M \mid f \circ \alpha=0\right\}$ and $\varphi: M^{I} \rightarrow M^{J}$ is such that $\pi_{f} \circ \varphi=f$ for every $f \in J$, where $\pi_{f}: M^{J} \rightarrow M$ is the canonical projection, then $\left(k_{\alpha}, K_{\alpha}\right)$ is the kernel of $\varphi$ and therefore $K_{\alpha} \in$ Copres $(M)$. 
In particular, $\sigma$ factors as $A \stackrel{\lambda_{\sigma}}{\rightarrow} K_{\sigma} \stackrel{k_{\sigma}}{\rightarrow} M^{H}$ with $K_{\sigma} \in \operatorname{Copres}(M)$, and we shall show that $\lambda_{\sigma}$ is a Copres $(M)$-preenvelope. To see this, let $\beta: A \rightarrow F$ be any morphism in $\mathcal{A}$ with $F \in \operatorname{Copres}(M)$ and take an exact sequence

$$
0 \rightarrow F \stackrel{v}{\rightarrow} M^{I} \stackrel{g}{\rightarrow} M^{X}
$$

in $\mathcal{A}$. Now set $\alpha=v \circ \beta: A \rightarrow M^{I}$ and consider the factorization $A \stackrel{\lambda_{\alpha}}{\rightarrow} K_{\alpha} \stackrel{k_{\alpha}}{\rightarrow} M^{I}$ of $\alpha$ given by the above claim. If $q_{x}: M^{X} \rightarrow M$ are the canonical projections, then we get

$$
(v, F)=\cap\left\{\operatorname{Ker}\left(q_{x} \circ g\right) \mid x \in X\right\} .
$$

Now, since $q_{x} \circ g \circ \alpha=q_{x} \circ g \circ v \circ \beta=0$ for each $x \in X$, we see that $k_{\alpha}$ factors through $v$ and therefore $\beta$ factors through $\lambda_{\alpha}$. So, in order to see that $\beta$ factors through $\lambda_{\sigma}$ it is enough to see that $\lambda_{\alpha}$ does. To prove this, let $J$ and $\varphi: M^{I} \rightarrow M^{J}$ be as in the proof of the above claim and consider the diagram

$$
\begin{aligned}
& A \stackrel{\lambda_{\sigma}}{\longrightarrow} K_{\sigma} \stackrel{k_{\sigma}}{\longrightarrow} M^{H} \\
& \text { ॥ } \quad \downarrow g^{\prime} \quad \downarrow g \\
& A \stackrel{\lambda_{\alpha}}{\longrightarrow} K_{\alpha} \stackrel{k_{\alpha}}{\longrightarrow} M^{I} \stackrel{\varphi}{\longrightarrow} M^{J} \text {. }
\end{aligned}
$$

Since $\sigma$ is a $\operatorname{Prod}(M)$-envelope, there exists $g: M^{H} \rightarrow M^{I}$ such that $\alpha=g \circ \sigma$. But now the morphism $\varphi \circ g: M^{H} \rightarrow M$ verifies $\varphi \circ g \circ \sigma=\varphi \circ \alpha=0$, and therefore $\varphi \circ g \circ k_{\sigma}=0$ by the definition of $k_{\sigma}$; this implies that $g \circ k_{\sigma}$ factors through $k_{\alpha}$, i.e. there exists $g^{\prime}: K_{\sigma} \rightarrow K_{\alpha}$ with $g \circ k_{\sigma}=k_{\alpha} \circ g^{\prime}$, and then it is clear that $\lambda_{\alpha}=g^{\prime} \circ \lambda_{\sigma}$, which is the factorization we were seeking.

Next we list several sufficient conditions for Copres $(M)$ to be reflective in $\mathcal{A}$.

Proposition 4.4. Let $\mathcal{A}$ be a well-powered complete abelian category with enough injectives, and consider the following conditions on an object $M$ of $\mathcal{A}$.

(a) $M$ has injective dimension at most 1 and $\operatorname{Ext}^{1}\left(M^{I}, M\right)=0$ for every set $I$.

(b) $\operatorname{Ext}^{1}(C, M)=0$ for each $C \in \operatorname{Cogen}(M)$.

(c) Hom $(-, M)$ takes exact sequences of the form $0 \rightarrow A \rightarrow M^{I} \rightarrow C \rightarrow 0$ with $C \in \operatorname{Cogen}(M)$ to exact sequences in $\mathrm{Ab}$.

(d) Cogen $(M)$ is closed for extensions.

(e) Copres $(M)$ is a reflective subcategory of $\mathcal{A}$.

Then $(\mathrm{a}) \Rightarrow(\mathrm{b}) \Rightarrow(\mathrm{c}) \Rightarrow(\mathrm{e})$ and $(\mathrm{b}) \Rightarrow(\mathrm{d}) \Rightarrow(\mathrm{e})$.

Proof. $\quad(\mathrm{a}) \Rightarrow(\mathrm{b})$. Let $\quad C \in \operatorname{Cogen}(M)$ and take an exact sequence $0 \rightarrow C \rightarrow M^{I} \rightarrow B \rightarrow 0$; now we have $\operatorname{Ext}^{1}\left(M^{I}, M\right)=0=\operatorname{Ext}^{2}(B, M)$, and so $\operatorname{Ext}^{1}(C, M)=0$.

(b) $\Rightarrow(\mathrm{c})$. This is clear.

(c) $\Rightarrow(\mathrm{e})$. It is clearly enough to show that, if $B \in \operatorname{Copres}(M)$, then the kernel $A$ of any morphism $f: B \rightarrow M^{I}$ is also in Copres $(M)$. Since

$$
A=\cap\{\operatorname{Ker} h \mid h \in \operatorname{Hom}(B, M) \text { with } A \subseteq \operatorname{Ker} h\},
$$

by taking a copresentation $0 \rightarrow B \stackrel{v}{\rightarrow} M^{J} \rightarrow M^{K}$, the hypothesis implies that 


$$
A=\cap\left\{\operatorname{Ker}(g \circ v) \mid g \in \operatorname{Hom}\left(M^{J}, M\right) \text { with } A \subseteq \operatorname{Ker}(g \circ v)\right\} .
$$

Taking now the composition $A \hookrightarrow B \stackrel{v}{\rightarrow} M^{J}$ and $L=\left\{g \in \operatorname{Hom}\left(M^{J}, M\right) \mid\right.$ $A \subseteq \operatorname{Ker}(g \circ v)\}$, we get an exact sequence $0 \rightarrow A \rightarrow M^{J} \rightarrow M^{L}$ which proves the claim.

(b) $\Rightarrow(\mathrm{d})$. Let $0 \rightarrow A \rightarrow B \rightarrow C \rightarrow 0$ be exact in $\mathcal{A}$ with $A, C \in \operatorname{Cogen}(M)$; taking the pushout of $A \rightarrow B$ and a monomorphism $A \rightarrow M^{I}$, we get a commutative diagram with exact rows and vertical monomorphisms:

$$
\begin{aligned}
& 0 \rightarrow A \longrightarrow B \rightarrow C \rightarrow 0 \\
& \downarrow \quad \downarrow \quad \| \\
& 0 \rightarrow M^{I} \rightarrow \bar{B} \rightarrow C \rightarrow 0
\end{aligned}
$$

Now the lower row splits, so that $\bar{B}$, and hence $B$, are in Cogen $(M)$.

(d) $\Rightarrow(\mathrm{e})$. It is enough to show that, if $0 \rightarrow A \rightarrow B \rightarrow C \rightarrow 0$ is exact in $\mathcal{A}$ with $B \in \operatorname{Copres}(M)$ and $C \in \operatorname{Cogen}(M)$, then $A \in \operatorname{Copres}(M)$. For, we have an exact sequence $0 \rightarrow B \rightarrow M^{I} \rightarrow D \rightarrow 0$ with $D \in \operatorname{Cogen}(M)$, and applying the cross lemma to these sequences we get new exact sequence $0 \rightarrow C \rightarrow E \rightarrow D \rightarrow 0$ (and so, by (d),

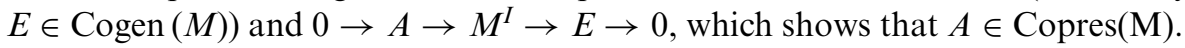

EXAmple 4.1. (1) None of the non-stated implications in the above result is true. For instance, if $M$ is a cogenerator of $\mathcal{A}$ then (d) and (e) trivially hold, while (b) and (c) hold only if $M$ is injective. On the other hand, if $S$ is a simple module which is finite dimensional over its endomorphism ring, then Cogen $(S)$ consists of the semisimple $S$-homogenous modules (cf. [23, Proposition 4.5]), and thus (c) and (e) hold, while (b) and (d) hold only if $\operatorname{Ext}^{1}(S, S)=0$, which is not always the case (e.g. take $S=\mathbb{Z}_{2}$ as a $\mathbb{Z}$-module). Finally, if $S$ is as above and $\operatorname{Ext}^{1}(S, S)=0$ then (b) holds, but $S$ need not have injective dimension $\leq 1$.

(2) If $M$ is an injective object of $\mathcal{A}$ then condition (a) in Proposition 4.4 holds, and hence Copres $(M)$ is reflective in $\mathcal{A}$. In that case, $\mathcal{T}=\{T \in \mathcal{A} \mid \operatorname{Hom}(T, M)=0\}$ is a localizing subcategory of $\mathcal{A}$ and Copres $(M)$ is just the subcategory of $\mathcal{T}$-closed objects in the terminology of Gabriel [14]. Moreover, the left adjoint $a: \mathcal{A} \rightarrow \operatorname{Copres}(M)$ of the inclusion is exact and identifies Copres $(M)$ with the quotient category $\mathcal{A} / \mathcal{T}$.

(3) A typical class of objects satisfying condition (a) in Proposition 4.4 is that of partial cotilting objects, i.e. objects $M$ such that $\operatorname{Cogen}(M) \subseteq \operatorname{Ker}_{\operatorname{Ext}^{1}}(-, M)$ and $\operatorname{Ker}_{\operatorname{Ext}}{ }^{1}(-, M)$ is a hereditary torsion class (cf. the obvious generalization of [7, Lemma 2.6(b)]).

(4) If $M_{R}$ is a quasi-injective module which is finitely generated over its endomorphism ring $S$ then, by [6, Lemma 1.5], $M^{I}$ is $M$-generated for every set $I$. But then condition (c) in Proposition 4.4 holds since the exact sequences under consideration are in the category $\sigma[M]$ of $M$-subgenerated modules (see [35, (16.1)]). Therefore Copres $(M)$ is a reflective subcategory of $\operatorname{Mod}_{R}$. If ${ }_{S} M$ is not finitely generated and we denote by $\tau_{M}(N)$ the trace of $M$ in $N$, the $M$-copresented objects in $\sigma[M]$ are those $X$ fitting in an exact sequence of the form $0 \rightarrow X \rightarrow \tau_{M}\left(M^{I}\right) \rightarrow$ $\tau_{M}\left(M^{J}\right)$ for some sets $I, J$. They form a reflective subcategory of $\sigma[M]$.

(5) In general, not even for $\mathcal{A}=\operatorname{Mod}_{R}$ it is true that Copres $(M)$ is a reflective subcategory of $\mathcal{A}$, for a given object $M$. To show this we give the following proposition. 
Proposition 4.5. The following assertions are equivalent for an artinian commutative ring $R$ :

(a) $R$ is quasi-Frobenius (i.e. self-injective);

(b) Copres $(R)$ is a reflective subcategory of $\operatorname{Mod}_{R}$;

(c) Copres $(R)$ is closed for direct summands.

Proof. The implications (a) $\Rightarrow(\mathrm{b}) \Rightarrow(\mathrm{c})$ are clear. To see that $(\mathrm{c}) \Rightarrow(\mathrm{a})$, it is not restrictive to assume that $R$ is also local. Suppose $R$ is not quasi-Frobenius. Then the simple module $S$ is not reflexive [1, (30.8)] and thus, by [21, Exercise (12), p. 331], we have $\operatorname{Soc}(S) \cong S^{n}$ for some integer $n \geq 2$ and $S^{*}=\operatorname{Hom}_{R}(S, R) \cong S^{n}$. Now, by using the fact that $R^{I}$ is projective for each set $I$, one easily proves that any non-zero finitely generated module of Copres $(R)$ must be isomorphic to $X^{*}$ for some nonzero finitely generated $R$-module $X$. On the other hand, such an $X^{*}$ contains a copy of $S^{*}$, and thus it cannot be isomorphic to $S$. Hence $S^{n} \cong S^{*} \in \operatorname{Copres}(R)$ and $S \notin \operatorname{Copres}(R)$.

REMARK 4.2. All results about Copres $(M)$ admit straightforward dualizations to a co-complete abelian category $\mathcal{A}$. Hence, the subcategory Pres $(M)$ of $M$-presented objects is coreflective in $\mathcal{A}$ if and only if it is closed for cokernels. When $\mathcal{A}=\operatorname{Mod}_{R}$ that includes, as particular situations, the cases when $M_{R}$ is tilting [17], $M_{R}$ is $\Sigma$-quasiprojective [16] or, more generally, $M_{R}$ is $w$ - $\Sigma$-quasiprojective in the sense of [6].

5. Reflectivity of $\operatorname{Add}(\boldsymbol{M})$. An additive category $\mathcal{C}$ will be called a generalized Krull-Schmidt category when every object in $\mathcal{C}$ is a direct sum of indecomposable objects with local endomorphism ring and, moreover, the isomorphism classes of indecomposable objects form a set. In this section, we study reflective generalized Krull-Schmidt subcategories of $\operatorname{Mod}_{R}$. It is clear (see [23]) that it is equivalent to study reflective subcategories of the form $\operatorname{Add}\left(M_{R}\right)$ (i.e., the subcategory of direct summands of coproducts of copies of a module $\left.M_{R}\right)$.

By [23], Add $(M)$ is closed for products if and only if it is covariantly finite in $\operatorname{Mod}_{R}$, in which case $M$ is called product-complete. Such a module is always $\Sigma$-pureinjective, so that Add $(M)$ consists in that case of the direct summands of products of copies of $M_{R}$. In particular, $\operatorname{Add}(M) \subseteq \operatorname{Copres}(M)$, and the converse inclusion holds exactly when $\operatorname{Add}(M)$ is reflective in $\operatorname{Mod}_{R}$.

Proposition 5.1. Let $M_{R}$ be any module. Then the following assertions are equivalent:

(a) $\operatorname{Add}(M)$ is reflective in $\operatorname{Mod}_{R}$;

(b) $\operatorname{Add}(M)$ is closed for products and kernels;

(c) $\operatorname{Add}(M)=\operatorname{Copres}(M)$.

Proof. (a) $\Longleftrightarrow$ (b). Follows from Theorem 3.1 and [23, Theorem 3.1].

(b) $\Rightarrow$ (c). Condition (b) implies Copres $(M) \subseteq \operatorname{Add}(M)$, and hence equality.

(c) $\Rightarrow$ (a). We have to show that $\operatorname{Add}(M)=\operatorname{Copres}(M)$ is closed for kernels, and it is enough to see that, if $0 \rightarrow A \rightarrow B \rightarrow C \rightarrow 0$ is exact with $B \in \operatorname{Copres}(M)$ and $C \in \operatorname{Cogen}(M)$, then $A \in \operatorname{Copres}(M)$. But we have a split sequence $0 \rightarrow B \rightarrow M^{I} \rightarrow$ $D \rightarrow 0$ with $D \in \operatorname{Cogen}(M)$, and by applying the cross lemma we get a split sequence 
$0 \rightarrow C \rightarrow X \rightarrow D \rightarrow 0$, whence $X \in \operatorname{Cogen}(M)$, and an exact sequence $0 \rightarrow A \rightarrow$ $M^{I} \rightarrow X \rightarrow 0$, which shows that $A \in \operatorname{Copres}(M)$.

For the rest of this section, we fix a nonzero module $M_{R}$ and set $S=\operatorname{End}\left(M_{R}\right)$ and $\bar{R}=\operatorname{BiEnd}\left(M_{R}\right)=\operatorname{End}\left({ }_{S} M\right)$. We write $\rho: R \rightarrow \bar{R}$ for the canonical ring homomorphism, and denote by $\rho *: \operatorname{Mod}_{\bar{R}} \rightarrow \operatorname{Mod}_{R}$ the restriction of scalars functor.

In the examples that will come, we will frequently consider the case when $M=R \oplus X$ for certain module $X_{R}$. Then there is a canonical ring isomorphism $S \cong\left(\begin{array}{cc}R & \operatorname{Hom}_{R}(X, R) \\ X & \text { End }\left(X_{R}\right)\end{array}\right)$ and, setting $e=\left(\begin{array}{ll}1 & 0 \\ 0 & 0\end{array}\right) \in S$, we have ${ }_{S} M \cong S e$ and thus $\bar{R} \cong e S e \cong R$.

The proof of the following lemma is left to the reader.

Lemma 5.2. The functor $\rho *$ induces an equivalence of categories $\operatorname{Add}\left(M_{\bar{R}}\right) \cong$ $\operatorname{Add}\left(M_{R}\right)$.

LeMmA 5.3. $M_{R}$ is product complete if and only if $M_{\bar{R}}$ is product complete.

Proof. The "if" part is trivial. For the converse, fix a set $I$; by the preceding lemma there is a module in $\operatorname{Add}\left(M_{\bar{R}}\right)$ whose restriction of scalars is isomorphic to $M_{R}^{I}$. So we have a right $\bar{R}$-module structure on the abelian group $M^{I}$ such that $M^{I} \in \operatorname{Add}\left(M_{\bar{R}}\right)$. Now, by Lemma 5.2, the canonical projections $p_{i}: M^{I} \rightarrow M$ are $\bar{R}$ homomorphisms and hence, for any $m=\left(m_{i}\right)_{i \in I} \in M^{I}$ and $\alpha \in \bar{R}$, we have $p_{j}(m \alpha)=\left(p_{j} m\right) \alpha=m_{j} \alpha$ and therefore $\left(m_{i}\right)_{i \in I} \alpha=\left(m_{i} \alpha\right)_{i \in I}$. This means that the described structure of $M^{I}$ is the canonical one (induced by the natural structure on $M_{\bar{R}}$ ), and thus $M_{\bar{R}}$ is product-complete.

THEOREM 5.4. Add $\left(M_{R}\right)$ is reflective in $\operatorname{Mod}_{R}$ if and only if $\operatorname{Add}\left(M_{\bar{R}}\right)$ is reflective in $\operatorname{Mod}_{\bar{R}}$. When these conditions hold, we have:

(i) ${ }_{S} M$ is projective and finitely generated;

(ii) $\bar{R}$ is right $\Sigma$-pure-injective;

(iii) the canonical map $\rho: R_{R} \rightarrow \bar{R}_{R}$ is the $\operatorname{Add}\left(M_{R}\right)$-reflection of $R_{R}$.

Proof. If $\operatorname{Add}\left(M_{\bar{R}}\right)$ is reflective in $\operatorname{Mod}_{\bar{R}}$ then $M_{R}$ is product-complete by Lemma 5.3, and we only have to check that every $R$-homomorphism $f: X \rightarrow Y$ with $X, Y \in \operatorname{Add}\left(M_{R}\right)$ has its kernel in Add $(M)$. But Lemma 5.2 tells us that $f$ is also a $\bar{R}$ homomorphism. Hence $\operatorname{Ker} f \in \operatorname{Add}\left(M_{\bar{R}}\right)$ and, by restricting scalars, $\operatorname{Ker} f \in$ $\operatorname{Add}\left(M_{R}\right)$. For the converse, reverse the argument.

Suppose now that $\operatorname{Add}\left(M_{R}\right)$ is reflective in $\operatorname{Mod}_{R}$.

(i) Let $\lambda: R \rightarrow X$ be an $\operatorname{Add}(M)$-reflection; since $R_{R}$ is finitely generated, it is easy to see that $X$ is a direct summand of $M^{n}$ for some positive integer $n$. Now, the image of $\lambda$ by the functor $\operatorname{Hom}_{R}(-, M)$ is an isomorphism $\operatorname{Hom}_{R}(X, M) \cong M$ and it is clear that $\operatorname{Hom}_{R}(X, M)$ is projective and finitely generated as a left $S$-module.

(ii) Since $M_{\bar{R}}$ is product-complete, $\operatorname{Add}\left(M_{\bar{R}}\right)$ consists of $\Sigma$-pure-injective modules, and since $M_{\bar{R}}$ is a generator [34, (IV.6.7)] we have $\bar{R}_{R} \in \operatorname{Add}\left(M_{\bar{R}}\right)$.

(iii) Lemma 5.2 implies that $\bar{R}_{R} \in \operatorname{Add}\left(M_{R}\right)$, and it is easy to see that the composition of the natural maps

$$
M_{\bar{R}} \stackrel{\cong}{\longrightarrow} \operatorname{Hom}_{\bar{R}}(\bar{R}, M)=\operatorname{Hom}_{R}(\bar{R}, M) \stackrel{\operatorname{Hom}_{R}(\rho, M)}{\longrightarrow} \operatorname{Hom}_{R}(R, M) \stackrel{\cong}{\longrightarrow} M_{R}
$$


is the identity. Therefore $\operatorname{Hom}_{R}(\rho, M)$ is an isomorphism and it follows that $\rho$ is an $\operatorname{Add}\left(M_{R}\right)$-reflection.

REMARK 5.1. If $\operatorname{Add}\left(M_{R}\right)$ is reflective in $\operatorname{Mod}_{R}$ then $R \rightarrow \bar{R}$ is the ring of definable scalars corresponding to the definable subcategory $\operatorname{Add}\left(M_{R}\right)$ (see [22, Corollary 11.10]).

COROLlaRY 5.5. The following assertions are equivalent:

(a) $M_{R}$ is indecomposable and $\operatorname{Add}\left(M_{R}\right)$ is reflective in $\operatorname{Mod}_{R}$;

(b) $S$ is a division ring and ${ }_{S} M$ is finite dimensional.

Proof. (a) $\Rightarrow$ (b). From [23, Theorem 4.1], [8, Proposition 4.4] and our Theorem 5.4 , one gets that ${ }_{S} M$ is free of finite rank and $S$ is left artinian and local. Hence ${ }_{S} M_{\bar{R}}$ defines a Morita equivalence and $\operatorname{Add}\left(M_{\bar{R}}\right)=\operatorname{Proj}_{\bar{R}}$. Therefore $\operatorname{rgD}(S)=\operatorname{rgD}(\bar{R}) \leq 2$, which implies that $S$ is a division ring.

(b) $\Rightarrow$ (a). Since $\bar{R}$ is semisimple, the implication is clear.

A module $M_{R}$ is called self-small when, for every set $I$, the canonical homomorphism $\operatorname{Hom}_{R}(M, M)^{(I)} \rightarrow \operatorname{Hom}_{R}\left(M, M^{(I)}\right)$ is an isomorphism. It is clear that $M_{R}$ is self-small if and only if so is $M_{\bar{R}}$. We shall say that a ring $A$ is right proreflective when $\operatorname{Proj}_{A}$ is reflective in $\operatorname{Mod}_{A}$, i.e., when $A$ is right perfect, left coherent and $\operatorname{rgD}(A) \leq 2$.

Proposition 5.6. Let $M_{R}$ be self-small. Then the following assertions are equivalent:

(a) $\operatorname{Add}\left(M_{R}\right)$ is reflective in $\operatorname{Mod}_{R}$;

(b) $S$ is right pro-reflective and ${ }_{S} M$ is finitely generated projective.

In the case when these conditions hold, we have the following results.

(i) $\bar{R}$ is right perfect.

(ii) $\operatorname{rgD}(R) \leq 2+\operatorname{pd}\left(M_{\bar{R}}\right)$.

(iii) Assume that $M_{\bar{R}}$ is finitely generated. Then $\bar{R}$ is left coherent if and only if $\operatorname{Hom}_{R}(M, \bar{R})$ is a finitely generated left $R$-module.

Proof. The equivalence of (a) and (b) is proven as Proposition 3.8 in [23], using Theorem 5.4 (i). For the rest, by replacing $M$ by some power $M^{n}$ if necessary, we can assume that $M=\bar{R} \oplus X$ for certain $X \in \operatorname{Mod}_{R}$.

(i) Since $S$ is right perfect and $S=\operatorname{End}(\bar{R} \oplus X) \cong\left(\begin{array}{cc}\bar{R} & \operatorname{Hom}(X, \bar{R}) \\ X & \operatorname{End}(X)\end{array}\right)$, we get that $\bar{R}$ is right perfect [1, (28.6)].

(ii) For each $N_{\bar{R}}$, we get an exact sequence $0 \rightarrow K \rightarrow P_{1} \rightarrow P_{0} \rightarrow N \rightarrow 0$ in $\operatorname{Mod}_{\bar{R}}$ with $P_{0}$ and $P_{1}$ projective. Since $M_{\bar{R}}$ is a generator, we have $\operatorname{Proj}_{\bar{R}} \subseteq \operatorname{Add}\left(M_{\bar{R}}\right)$, and therefore $K \in \operatorname{Add}\left(M_{\bar{R}}\right)$, which clearly implies that $\operatorname{pd}\left(K_{\bar{R}}\right) \leq \operatorname{pd}\left(M_{\bar{R}}\right)$, whence the result follows.

(iii) Assume that $M_{\bar{R}}$ is finitely generated and (a), (b) hold, so that $\bar{R}$ is right perfect. If $\bar{R}$ is left coherent then it is indeed strongly left coherent (or left $\Pi$-coherent) in the sense of $[\mathbf{1 3}]$, and therefore $\operatorname{Hom}(M, \bar{R})$ is a finitely generated left $\bar{R}$ module by [5, Theorem 1].

Conversely, suppose that $\operatorname{Hom}(M, \bar{R})$ is a finitely generated left $\bar{R}$-module. Considering $S$ as matrices in the above sense, we consider the element 
$e=\left(\begin{array}{ll}1 & 0 \\ 0 & 0\end{array}\right) \in S$ and the bimodule ${ }_{\bar{R}}(e S)_{S}$. In ${ }_{\bar{R}}$ Mod we have $e S \cong$ $\bar{R} \oplus \operatorname{Hom}_{R}(X, \bar{R})$, and since this is finitely generated by the hypothesis, [6, Lemma 1.5] says that $(e S)^{I}$ is $e S$-generated in $\operatorname{Mod}_{S}$. Now, since the functor $\operatorname{Hom}_{S}(e S,-): \operatorname{Mod}_{S} \rightarrow \operatorname{Mod}_{\bar{R}}$ takes projective $e S$-generated right $S$-modules to projective right $\bar{R}$-modules, we infer that $\bar{R}^{I} \cong \operatorname{Hom}_{S}\left(e S,(e S)^{I}\right)$ is projective in $\operatorname{Mod}_{\bar{R}}$, so that $\bar{R}$ is left coherent.

Example 5.1. (1) In the situation of Proposition 5.6 we can have $\operatorname{rgD}(\bar{R})=\infty$ and $\bar{R}$ not left coherent. Indeed, let us consider a non-Artinian commutative local ring $R$ with $J^{2}=0$, where $J$ is the radical (e.g. $R=\frac{K\left[x_{1}, \ldots x_{n}, \ldots\right]}{\left(x_{1}, \ldots x_{n}, \ldots\right)^{2}}$ with $K$ a field). We propose the reader to prove that, if $\left\{e_{1}, \ldots, e_{t}\right\}$ is a finite family of elements in $R^{I}$ (for some set $I$ ) which is $R / J$-linearly independent modulo $J^{I}$, then it is $R$-linearly independent and thus $\sum_{i=1}^{t} e_{i} R=\oplus_{i=1}^{t} e_{i} R$ is a projective submodule of $R^{I}$. Once this is done, if $\left\{e_{\alpha} \mid \alpha \in A\right\}$ is a maximal family of $R / J$-linearly independent elements of $R^{I}$ modulo $J^{I}$ and $\left\{f_{\beta} \mid \beta \in B\right\}$ is a maximal family of $R / J$-linearly independent elements of $J^{I}$ modulo $R^{I} J$, then one easily sees that $R^{I}=\left(\oplus e_{\alpha} R\right) \oplus\left(\oplus f_{\beta} R\right)$, so that $R^{I}$ is a direct sum of a free and a semisimple submodule. If now $M=R \oplus R / J$, then $\operatorname{Add}(M)$ is clearly reflective in $\operatorname{Mod}_{R}$, but $\bar{R} \cong R$ has infinite global dimension and is not coherent.

(2) We do not know if a module $M_{R}$ satisfying either (a) or (b) in Proposition 5.6 is necessarily self-small, but it need not be finitely generated over $\bar{R}$. Indeed, if $R$ is the Kronecker algebra over a field $K$, then the generic module $G_{R}$ (cf. [20]) satisfies End $\left(G_{R}\right) \cong K(X)$ and $\operatorname{dim}_{K(X)} G=2$. So $G_{\operatorname{BiEnd}(G)}$, and consequently $G_{R}$, is self small. Now set $M=R \oplus G$, so that $\bar{R}=R$ and $M_{R}$ is not finitely generated; since $\operatorname{Hom}(G, R)=0$, it follows easily that (a-b) in Proposition 5.6 hold for $M$.

(3) In Proposition 5.6, it is easy to give examples where both $S$ and $\bar{R}$ are right pro-reflective but $M_{R}$ is not an endogenerator. For instance, take any right proreflective ring $R$ and a module $X_{R}$ such that $D=\operatorname{End}\left(X_{R}\right)$ is a division ring and ${ }_{D} X$ is finite-dimensional (if $R$ is a $C$-algebra such that $R / J(R)$ is an Artin $C$-algebra, then every simple right $R$-module has that property). If $X_{R}$ is not a direct summand of any kernel of a morphism between projectives, then $M=R \oplus X$ satisfies all the above conditions.

In what follows, we shall deal with the special case in Theorem 5.4 when ${ }_{S} M$ is a (pro)generator. For this purpose we make the following definition.

Definition 5.1. A module $M_{R}$ will be called a reflective endogenerator when $\operatorname{Add}\left(M_{R}\right)$ is reflective in $\operatorname{Mod}_{R}$ and ${ }_{S} M$ is a generator. Two reflective endogenerators $M_{R}$ and $N_{R}$ will be called equivalent when $\operatorname{Add}\left(M_{R}\right)=\operatorname{Add}\left(N_{R}\right)$.

In this situation $M_{\bar{R}}$ is also a progenerator, so that $\operatorname{Add}\left(M_{\bar{R}}\right)=\operatorname{Proj}_{\bar{R}}$ and $S$ and $\bar{R}$ are Morita equivalent rings. By Proposition 5.6, both rings are right pro-reflective. On the other hand, since direct summands of $M_{R}$ and $M_{\bar{R}}$ are the same, we have $M=M_{1}^{n_{i}} \oplus \ldots \oplus M_{t}^{n_{t}}$, where the $n_{i}$ are positive integers and $M_{1}, \ldots, M_{t}$ are pairwise non-isomorphic indecomposable modules.

Definition 5.2. A ring homomorphism $\varphi: R \rightarrow A$ will be called a right pseudoepimorphism when the canonical ring homomorphism $A \cong \operatorname{End}\left(A_{A}\right) \rightarrow \operatorname{End}\left(A_{R}\right)$ 
induced by $\varphi^{*}$ is onto (and hence, an isomorphism). Two right pseudoepimorphisms $\varphi: R \rightarrow A$ and $\varphi^{\prime}: R \rightarrow A^{\prime}$ will be said to be equivalent when there is a ring isomorphism $\phi: A \rightarrow A^{\prime}$ such that $\phi \circ \varphi=\varphi^{\prime}$.

If $\varphi: R \rightarrow A$ is a right pseudoepimorphism then $A=\operatorname{BiEnd}\left(A_{R}\right)$ and $\varphi$ is (equivalent to) the canonical map $R \rightarrow \operatorname{BiEnd}\left(A_{R}\right)$; by Proposition 5.6, $A_{R}$ is a reflective endogenerator if and only if $A$ is right pro-reflective. In fact, we have:

Proposition 5.7. Let $R$ be any ring. There is a bijective correspondence between:

(a) the set of equivalence classes of reflective endogenerators in $\operatorname{Mod}_{R}$; and

(b) the set of equivalence classes of right pseudoepimorphisms $\varphi: R \rightarrow A$ with $A$ right pro-reflective.

Proof. If $\operatorname{Add}\left(M_{R}\right)$ is reflective then $\bar{R}_{\bar{R}} \in \operatorname{Add}\left(M_{\bar{R}}\right)$ by Theorem 5.4, and End $\left(\bar{R}_{\bar{R}}\right)=\operatorname{End}\left(\bar{R}_{R}\right)$ by Lemma 5.2 , so that the ring homomorphism $\rho: R \rightarrow \bar{R}$ is a right pseudoepimorphism. Note that, if $\operatorname{Add}\left(M_{R}\right)=\operatorname{Add}\left(N_{R}\right)$, then $\operatorname{BiEnd}\left(M_{R}\right)=\operatorname{BiEnd}\left(N_{R}\right)[1,(14.1)]$, and thus $\rho$ does not depend on $M_{R}$ but on its equivalence class. If, moreover, $M_{R}$ is a reflective endogenerator, then $\bar{R}$ is right proreflective and thus the assignation $M_{R} \mapsto \rho$ establishes a map from (a) to (b).

Conversely, if $\varphi: R \rightarrow A$ is as in (b), then $\operatorname{Add}\left(A_{R}\right)$ is reflective in $\operatorname{Mod}_{R}$ by the remark above, so that $A_{R}$ is a reflective endogenerator in $\operatorname{Mod}_{R}$. Clearly, the assignation $\varphi \mapsto A_{R}$ depends only on the equivalence class of $\varphi$, and so it establishes a map from (b) to (a) which is an inverse for $M_{R} \mapsto \rho$.

REMARK 5.2. (1) If $\varphi$ is a right pseudoepimorphism then the restriction of $\varphi_{*}$ to $\operatorname{Add}\left(A_{A}\right)$ is a full functor. In particular, when the $\operatorname{ring} A$ is semisimple, every pseudoepimorphism $R \rightarrow A$ is an epimorphism (cf. [34, (XI.1.2)]). Thus, in view of Corollary 5.5, the above proposition extends Proposition 2.1 in [27].

(2) The bijection of Proposition 5.7 induces another one between the set of: (a) equivalence classes of flat reflective endogenerators; and (b) isomorphism classes of flat ring epimorphisms $\varphi: R \rightarrow A$ with $A$ right pro-reflective. Indeed, if $M_{R}$ is a flat reflective endogenerator then every module in $\operatorname{Add}\left(M_{R}\right)$ (and in particular $\bar{R}_{R}$ ) is flat, and thus all we need prove is that a flat right pseudoepimorphism $\varphi: R \rightarrow A$ with $A$ right pro-reflective is an epimorphism. But, in that case, $A \oplus_{R} A$ is projective (and hence torsionless) in $\operatorname{Mod}_{A}$ and the multiplication map $\mu: A \oplus_{R} A \rightarrow A$ is carried to an isomorphism by the functor $\operatorname{Hom}_{A}(-, A)$, so that it is an isomorphism and therefore $\varphi$ is a ring epimorphism by [34, (XI.1.2)].

(3) When the ring $R$ is commutative, there is a bijective correspondence between the sets of: (a) equivalence classes of reflective endogenerators in $\operatorname{Mod}_{R}$; (b) equivalence classes of ring epimorphisms $\varphi: R \rightarrow A$ where $A$ is a finite direct product of fields; and (c) finite parts of $\operatorname{Spec}(R)$. Indeed, one proves along the lines of [32, Corollary 1.2] that if $R \rightarrow A$ is a right pseudoepimorphism then the commutativity of $R$ implies that of $A$, so that pro-reflectivity of $A$ means that it is a finite direct product of fields. The rest follows the pattern of [27, Example 1.2.a]. The bijection (b) $\leftrightarrow$ (c) takes $\varphi: R \rightarrow K_{i} \times \ldots \times K_{n}$ to $\left\{\operatorname{Ker}\left(\pi_{i} \circ \varphi\right) \mid i=1, \ldots, n\right\}$ (where $\pi_{i}: \prod K_{i} \rightarrow K_{i}$ is the canonical projection) in one direction and $\left\{P_{1}, \ldots, P_{n}\right\}$ to $R \rightarrow Q\left(R / P_{1}\right) \times \ldots \times Q\left(R / P_{n}\right)$ (where $Q(D)$ is the quotient field of the domain $\left.D\right)$ in the other. 
EXAMPLE 5.2. (1) Let $D$ be a commutative integral domain with quotient field $Q$; then the inclusion $R=\left(\begin{array}{cc}D & D \\ 0 & D\end{array}\right) \hookrightarrow\left(\begin{array}{cc}Q & Q \\ 0 & Q\end{array}\right)=T$ is a ring epimorphism and, since $T$ is left and right hereditary and artinian, $T_{R}$ and ${ }_{R} T$ are both reflective endogenerators.

(2) Let $R$ be a right artinian ring and let $M_{R}$ be such that $\operatorname{Hom}_{R}(-, M)$ is an object of finite length in the category $\left(\bmod _{R}^{\text {op }}, \mathrm{Ab}\right)$. Let $\mathcal{I}_{M}$ be a family of representatives of the isomorphism classes of indecomposable modules $X \in \bmod _{R}$ with $\operatorname{Hom}_{R}(X, M) \neq 0$; by [3, Theorem 2.12] we know that $\mathcal{I}_{M}$ is finite, and we set $\tilde{M}=\bigoplus_{X \in \mathcal{I}_{M}} X$.

We claim that $\operatorname{Add}(\tilde{M})$ is reflective in $\operatorname{Mod}_{R}$, but $\tilde{M}$ is an endogenerator if and only if $\tilde{M}_{R}$ is projective. To prove that we first replace $M_{R}$ by $\tilde{M}_{R}$ if necessary and assume from the beginning that, for an indecomposable $X \in \bmod _{R}$, $\operatorname{Hom}_{R}(X, M) \neq 0$ implies $X \in \operatorname{Add}\left(M_{R}\right)$. In particular, if $\epsilon: P \rightarrow M$ is the projective cover of $M_{R}$ then $P \in \operatorname{Add}\left(M_{R}\right)$ and so the class of indecomposable projective direct summands of $M_{R}$ is not empty. We pick a set $\left\{P_{1}=e_{1} R, \ldots, P_{t}=e_{t} R\right\}$ of representatives of the isomorphism classes of these direct summands, where $\left\{e_{1}, \ldots, e_{t}\right\}$ are pairwise orthogonal idempotents of $R$. One immediately gets that, if $S=\operatorname{End}\left(M_{R}\right)$, then ${ }_{S} M=S e_{1} \oplus \ldots \oplus S e_{t}$ (viewing the $e_{i}$ as elements of $M_{R}$, since $e_{i} R$ is a direct summand of $M_{R}$ for each $\left.i=1, \ldots, t\right)$. Also, clearly, $S e_{i} \cong S \epsilon_{i}$, where $\epsilon_{i}$ is the idempotent endomorphism $M_{R} \rightarrow e_{i} R \rightarrow M_{R}$. Hence ${ }_{S} M$ is finitely generated projective. On the other hand, again by [3, Theorem 2.12], $S$ is left artinian and so $M_{R}$ has finite endolength, which implies that $\operatorname{Add}\left(M_{R}\right)$ is closed for products and direct limits (cf. [23, Theorem 4.1]).

If now $f: M^{(I)} \rightarrow M^{(J)}$ is a $R$-homomorphism, then it is the direct limit of a system of morphisms $f_{\alpha}: M^{n_{\alpha}} \rightarrow M^{(J)}$, whose kernels are in $\operatorname{Add}\left(M_{R}\right)$ by our choice of $M_{R}$. Hence $\operatorname{Ker} f=\lim \operatorname{Ker} f_{\alpha}$ is in $\operatorname{Add}\left(M_{R}\right)$ and therefore $\operatorname{Add}\left(M_{R}\right)$ is reflective in $\operatorname{Mod}_{R}$. Moreover, ${ }_{S} M$ is a generator if and only if all indecomposable direct summands of ${ }_{S} S$ appear as direct summands of ${ }_{S} M$. This is equivalent to saying that all indecomposable direct summands of $M_{R}$ belong, up to isomorphism, to the family $\left\{P_{1}, \ldots, P_{t}\right\}$, i.e., that $M_{R}$ is projective.

In view of the previous example, a natural question arises. Suppose that $\operatorname{Add}\left(M_{R}\right)$ is reflective in $\operatorname{Mod}_{R}$; are there only finitely many non-isomorphic indecomposable direct summands of $M_{R}$ ? Note that if $R$ is right pure-semisimple and $M_{R}$ is the direct sum of all indecomposable right $R$-modules, an affirmative answer would solve the "pure-semisimple conjecture" (see [19] and [33] for the most recent advances). Hence, the question seems difficult and we can only give the following partial affirmative answers.

Proposition 5.8. Let $R$ be a principal ideal domain, $Q$ its quotient field and $M$ an arbitrary $R$-module. Then $\operatorname{Add}\left(M_{R}\right)$ is reflective in $\operatorname{Mod}_{R}$ if and only if there are finitely many (and perhaps none) pairwise nonassociated prime elements $p_{1}, \ldots, p_{t}$ in $R$, integers $n_{1}, \ldots, n_{t} \geq 1$ and sets $I_{11}, \ldots, I_{1 n_{1}}, \ldots, I_{t 1}, \ldots, I_{t n_{t}}, J$ (non-empty except perhaps $J$ ) such that

$$
M \cong\left(\frac{R}{\left(p_{1}\right)}\right)^{\left(I_{11}\right)} \oplus \ldots \oplus\left(\frac{R}{\left(p_{1}^{n_{1}}\right.}\right)^{\left(I_{1 n_{1}}\right)} \oplus \ldots \oplus\left(\frac{R}{\left(p_{t}\right)}\right)^{\left(I_{t 1}\right)} \oplus \ldots \oplus\left(\frac{R}{\left(p_{t}^{n_{t}}\right)}\right)^{\left(I_{t n_{t}}\right)} \oplus Q^{(J)} .
$$


Proof. Assume first that $M$ has the given form; then by [23, Theorem 4.1] each summand is product-complete and hence so is $M$; since $\operatorname{Add}(M)$ is clearly closed for kernels, it is reflective in $\operatorname{Mod}_{R}$.

For the converse we can assume that $M$ is of the form $M=\oplus M_{\alpha}$ for some set $\left\{M_{\alpha} \mid \alpha \in A\right\}$ of pairwise non-isomorphic indecomposable $\Sigma$-pure-injective $R$-modules. Now, by [20, (8.56)], the indecomposable $\Sigma$-pure-injective $R$-modules are: (a) the quotient field $Q$; and, for every prime element $p \in R$, $\left(\mathrm{b}_{1}\right)$ the modules $R /\left(p^{n}\right)$ with $n \geq 1$; and $\left(\mathrm{b}_{2}\right)$ the Prüfer module $R /\left(p^{\infty}\right)$ (the $p$-adic module $\hat{R}_{p}$ is not $\Sigma$-pureinjective since it is not an artinian ring). Note that, for a fixed prime $p, \operatorname{Add}(M)$ cannot contain a copy of $R /\left(p^{n}\right)$ for each $n \geq 1$, since $\operatorname{Add}(M)$ is closed for inverse limits and it does not contain $\hat{R}_{p}$. On the other hand, the fact that $\operatorname{Add}(M)$ is closed for kernels implies that, if $R /\left(p^{n}\right)$ is among the $M_{a}$, then so is $R /\left(p^{i}\right)$ for each $i=1,2, \ldots, n$. And it also implies that no $R /\left(p^{\infty}\right)$ is a direct summand of $M$, because otherwise $\operatorname{Add}(M)$ would contain every $R /\left(p^{n}\right)$. So, we only have to prove that the number of distinct $R /(p)$ that can appear among the $M_{a}$ is finite. But if $R /\left(p_{i}\right) \in \operatorname{Add}(M)$ for infinitely many primes $\left\{p_{1}, p_{2}, \ldots\right\}$ then we would have $\oplus_{i=1}^{\infty} R /\left(p_{i}\right) \in \operatorname{Add}(M)$, so that the pure monomorphism $\oplus_{i=1}^{\infty} R /\left(p_{i}\right) \rightarrow \Pi_{i=1}^{\infty} R /\left(p_{i}\right)$ would split.

Proposition 5.9. Let $R$ be a finite dimensional tame hereditary algebra over a field $K$ which is not representation-finite, and let $G$ be the generic module. If $\operatorname{Add}\left(M_{R}\right)$ is reflective in $\operatorname{Mod}_{R}$, then there are finitely generated indecomposable $R$-modules $X_{1}, \ldots, X_{t}$ and (possibly empty) sets $I_{1}, \ldots, I_{t}$ and $J$ such that

$$
M_{R} \cong X_{1}^{\left(I_{1}\right)} \oplus \ldots \oplus X_{t}^{\left(I_{t}\right)} \oplus G^{(J)}
$$

Proof. Following the terminology of [29], this is proved like Proposition 5.8 by taking $S, S[n] S[\infty], \hat{S}$ and $G$ instead of $R /(p), R /\left(p^{n}\right), R /\left(p^{\infty}\right), \hat{R}_{p}$ and $Q$, where $S$ is a simple regular module and $G$ is the generic module.

REMARK 5.3. Notice the difference between reflectivity of $\operatorname{Add}(M)$ in $\operatorname{Mod}_{R}$ and reflectivity of add $(M)$ in $\bmod _{R}$. If $R$ is a right artinian ring and $M_{R}$ is the direct sum of one copy of each indecomposable $R$-module $X_{R}$ such that $\operatorname{Hom}(-, X)$ is an object of finite length in $\left(\mathrm{Mod}_{R}^{\mathrm{op}}, \mathrm{Ab}\right)$ (cf. Example 5.2), then add $\left(M_{R}\right)$ is reflective in $\bmod _{R}$ (and hence $\overrightarrow{\operatorname{add}\left(M_{R}\right)}$ is reflective in $\operatorname{Mod}_{R}$ by Proposition 3.4). However, it is well known (cf. [28]) that, when $R$ is a hereditary finite dimensional algebra over a field that is not representation-finite, $M_{R}$ has infinitely many non-isomorphic direct summands. Then Proposition 5.9 says that $\operatorname{Add}\left(M_{R}\right)$ is not reflective in $\operatorname{Mod}_{R}$ when $R$ is tame and not representation-finite.

Acknowledgement. The second and third authors are supported by the DGES of Spain (PB96-0961-C02-02) and by the Fundación Séneca (Communidad Autónoma de la Región de Murcia, PB/16/FS/97).

\section{REFERENCES}

1. F. Anderson and K. Fuller, Rings and Categories of Modules, 2nd ed. (SpringerVerlag, 1992). 
2. J. Asensio Mayor and J. Martínez Hernández, On flat and projective envelopes, $J$. Algebra 160 (1993), 434-440. $269-310$

3. M. Auslander, Representation theory of Artin algebras II, Comm. Algebra 1 (1974),

4. H. Bernecker, Flatness and absolute purity; applying functor categories to ring theory, J. Algebra 44 (1977), 411-419.

5. V. Camillo, Coherence for polynomial rings, J. Algebra 132 (1990), 72-76.

6. R. Colpi and C. Menini, On the structure of*-modules, J. Algebra 158 (1993), 400-419.

7. R. Colpi, A. Tonolo and J. Trlifaj, Partial cotilting modules and the lattices induced by them, Comm. Algebra 25 (1997), 3225-3237.

8. W. Crawley-Boevey, Modules of finite length over their endomorphism rings, Representations of algebras and related topics, London Math. Soc. Lecture Notes Series 168, 127-184. (Cambridge University Press, 1992).

9. W. Crawley-Boevey, Locally finitely presented additive categories, Comm. Algebra 22 (1994), 1641-1674.

10. N. Ding, On envelopes with the unique mapping property, Comm. Algebra 24 (1996), $1459-1470$.

11. E. Enochs, Injective and flat covers, envelopes and resolvents, Israel J. Math. 39 (1981), 189-209.

12. E. Enochs, J. Martínez Hernández and A. del Valle, Coherent rings of finite weak global dimension, Proc. Amer. Math. Soc. 126 (1998), 1611-1620.

13. M. Finkel-Jones, Flatness and f-projectivity of torsion-free modules and injective modules, in Advances in Non-commutative Ring Theory, Lecture Notes in Mathematics, No. 951 (Springer-Verlag, 1982), 94-116.

14. P. Gabriel, Des catégories abéliennes, Bull. Soc. Math. France 90 (1962), 323-448.

15. P. Gabriel and F. Ulmer, Lokal präsentierbare Kategorien, Lecture Notes in Mathematics, No. 221 (Springer-Verlag, 1971).

16. J. L. García Hernández and J. L. Gómez Pardo, On endomorphism rings of quasiprojective modules, Math. Z. 196 (1987), 87-108.

17. D. Happel and C. Ringel, Tilted algebras, Trans. Amer. Math. Soc. 274 (1982), 399-443.

18. H. Herrlich and G. Strecker, Category Theory (Allyn and Bacon, 1973).

19. I. Herzog, A test for finite representation type, J. Pure and App. Algebra 95 (1994), $151-182$.

20. C. Jensen and H. Lenzing, Model Theoretic Algebra (Gordon and Breach, 1989).

21. F. Kasch, Modules and Rings (Academic Press, 1982).

22. H. Krause, The spectrum of a module category. Habilitationsschrift. Preprint 98-004, SFB 343 (Univ. Bielefeld, 1998).

23. H. Krause and M. Saorín, On minimal approximations of modules, in Contemp. Math. (Proc. AMS Conf., New trends in the representation theory of finite dimensional algebras, Seattle, 1997), 229 (1998), 227-236.

24. S. MacLane, Categories for the Working Mathematician (Springer-Verlag, 1971).

25. J. Martínez Hernández, Relatively flat envelopes, Comm. Algebra 14 (1986), 867-884.

26. J. Rada and M. Saorin, Rings characterized by (pre)envelopes and (pre)covers of their modules, Comm. Algebra 26 (1998), 899-912.

27. C. Ringel, The spectrum of a finite dimensional algebra, in Proc. Conference on Ring Theory, Antwerp 1978 (Dekker, 1979).

28. C. Ringel, Tame algebras and integral quradratic forms (Springer-Verlag, 1984).

29. C. Ringel, The Ziegler spectrum of a tame hereditary algebra, Preprint 97-035, SFB 343 (Univ. Bielefeld, 1997).

30. J. Roos, Locally noetherian categories and generalized strictly linearly compact rings. In Category Theory, Homology Theory and their applications II, Lecture Notes in Mathematics, No. 92 (Springer-Verlag, 1969), 197-277.

31. H. Schubert, Categories (Springer, 1972).

32. L. Silver, Noncommutative localization and applications, J. Alebra 7 (1967), 44-76.

33. D. Simson, An Artin problem for division ring extensions and the pure semisimplicity conjecture I, Arch. Math. (Basel) 66 (1996), 114-122.

34. B. Stenström, Rings of Quotients (Springer-Verlag, 1975).

35. R. Wisbauer, Foundations of Module and Ring Theory (Gordon and Breach, 1991). 